\title{
On the Solution of the Scalar-plus-compact Problem by Argyros and Haydon
}

András Zsák (University of Cambridge, UK)

Whether there is a Banach space on which every operator is a compact perturbation of a scalar multiple of the identity was one of the most famous, longstanding open problems in the theory of Banach spaces. It is known as the scalarplus-compact problem and it was solved by Spiros Argyros and Richard Haydon some 10 years ago by constructing a Banach space that has the scalar-plus-compact property. In recognition of this major contribution to mathematics, Argyros and Haydon were invited speakers at the 2018 ICM in Rio de Janeiro. Their achievement was spectacular, particularly as at the time no one expected a breakthrough. The solution of the scalar-plus-compact problem caught not just the mathematical community but also Argyros and Haydon by surprise.

\section{The origins}

If $X$ is an infinite-dimensional Banach space, what operators, i.e. continuous linear maps, on $X$ are there? The HahnBanach theorem provides a rich supply of continuous linear functionals: for every non-zero vector $x$, there is a continuous linear map $f: X \rightarrow \mathbb{R}$ such that $f(x)=1$. It follows that, for non-zero vectors $x$ and $y$, there is a continuous rank-1 operator $T: X \rightarrow X$ with $T(x)=y$ given by $T(z)=f(z) y$. By taking sums, we obtain a large supply of finite-rank operators on $X$, and the limit of a sequence of finite-rank operators is compact. Recall that an operator $T$ on $X$ is compact if for every bounded sequence $\left(x_{n}\right)$ in $X$, the sequence $\left(T x_{n}\right)$ has a convergent subsequence. Whether every compact operator is the limit of a sequence of finite-rank operators was another very famous open problem going back to Banach's book [9]. It was solved in the negative by Per Enflo in 1973 by constructing a Banach space that fails the so-called approximation property [12]. In this article, all Banach spaces under consideration have the approximation property, and so every compact operator can be expressed as the limit of a sequence of finite-rank operators. Assuming this for our space $X$, let us continue by asking if there are any non-compact operators on $X$. The answer is trivially 'yes': any non-zero multiple of the identity operator is non-compact since our space $X$ is infinite-dimensional. The construction of operators in this generality stops here: there seems to be no general method of finding operators that are not of the form $\lambda \operatorname{Id}+K$, where $K$ is a compact operator. In contrast, specific spaces, like Hilbert space, have very rich algebras of operators. In his famous list of open problems from 1976, Joram Lindenstrauss [26] began with the following question.
(Q.1) Does there exist an infinite-dimensional Banach space $X$ so that every operator $T: X \rightarrow X$ is of the form $T=$ $\lambda \mathrm{Id}+K$, where $K$ is compact?

Lindenstrauss goes on and lists some of the peculiar properties such a space $X$ would have to possess:

(i) $X$ is not isomorphic to its subspaces of finite codimension.

(ii) $X$ is indecomposable, i.e. every decomposition of $X$ as a direct sum $X=Y \oplus Z$ is trivial: either $Y$ or $Z$ is finitedimensional.

(iii) Every operator on $X$ has a non-trivial, proper, closed invariant subspace, i.e. if $T$ is an operator on $X$ then there is a closed subspace $Y$ of $X$ such that $Y \neq\{0\}, Y \neq X$ and $T(Y) \subset Y$.

The first property follows since every operator on $X$ is either compact or, being a compact perturbation of a non-zero multiple of the identity operator, Fredholm with index zero. The second property can be established as follows. If $T=\lambda \mathrm{Id}+K$ is a projection on $X$ then $\lambda$ must be 0 or 1 ; hence, either $T$ or Id $-T$ is a compact projection and thus either the range or the kernel of $T$ is finite-dimensional. The third property is far from being straightforward and follows from a theorem of Aronszajn and Smith [8], which states that every compact operator on a (complex) Banach space has a non-trivial, proper, closed invariant subspace.

Independently of the scalar-plus-compact problem, the three properties above lead to three questions, each asking for the existence or otherwise of a Banach space satisfying the given property. These questions themselves became very important in their own right. This is hardly surprising; after all, these are fairly basic structure-theory questions of the kind that arise in most areas of mathematics. Indeed, in most fields of research, an important aim is to classify the central objects of study, in our case Banach spaces, and to decompose a general object into simpler building blocks. This usually goes hand-in-hand with a similar study of the morphisms, in our case operators. A very simple example is the question of diagonalisability of matrices. For general Banach space operators, a more basic question is concerned with the existence of invariant subspaces.

The first two of these three questions were already answered in the 1990s. Tim Gowers solved the so-called Banach hyperplane problem [18] by constructing a Banach space not isomorphic to its hyperplanes, i.e. its closed subspaces of codimension 1. His space also satisfied the stronger property (i) above. Another major breakthrough of the 1990s was 
the solution by Tim Gowers and Bernard Maurey of the unconditional basic sequence problem [21]: they constructed a space that contains no subspace with an unconditional basis. An unconditional basis is an infinite coordinate system with lots of symmetries. A Banach space that has an unconditional basis has many operators and is thus far from the scalar-plus-compact property. As pointed out by Bill Johnson at the time, the proof of Gowers and Maurey showed a much stronger property of their space: it is indecomposable (property (ii) above) and, in fact, even hereditarily indecomposable, meaning that no closed, infinite-dimensional subspace is decomposable. The study of hereditarily indecomposable (HI) spaces has since become a very important area in the field. HI constructions also happen to play a major role in the solution of the scalar-plus-compact problem.

Concerning property (iii), it was already known to John von Neumann that compact operators on Hilbert space have invariant subspaces. For general Banach spaces, this was proved by Aronszajn and Smith, as mentioned above. Whether every operator on an arbitrary Banach space has non-trivial, proper, closed invariant subspaces remained a major unsolved problem for some time. The first counterexamples were produced by Charles Read [28] and Per Enflo [13]. Both were highly complex examples that were later simplified, strengthened and generalised. It is still an open problem whether operators on Hilbert space have invariant subspaces. Indeed, it may even be possible that every dual operator on a dual Banach space has an invariant subspace. It is therefore significant that there is a Banach space on which it is true that every operator has an invariant subspace. The Argyros-Haydon space is the first such example.

\section{$2 \quad$ History and ingredients}

Recall that a Banach space is said to be hereditarily indecomposable (HI) if every subspace of $X$ is indecomposable. The first example of an HI space is the space $\mathfrak{X}_{\mathrm{GM}}$ of Gowers and Maurey that solved the unconditional basic sequence problem. Studying the operators on $\mathfrak{X}_{\mathrm{GM}}$, Gowers and Maurey proved in [21] that every operator from a subspace of $\mathfrak{X}_{\mathrm{GM}}$ to the whole space is the sum of a scalar multiple of the inclusion operator and a strictly singular operator, which implies that $\mathfrak{X}_{\mathrm{GM}}$ is HI. (The reverse implication also holds for a complex HI space by a result of Valentin Ferenczi [14].) In particular, every operator on $\mathfrak{X}_{\mathrm{GM}}$ is a strictly singular perturbation of a scalar multiple of the identity. A strictly singular operator is one that is not an isomorphism on any (infinite-dimensional) subspace. Equivalently, an operator $T$ on a Banach space $X$ is strictly singular if every subspace contains for every $\varepsilon>0$ an element $x$ such that $\|T x\|<\varepsilon\|x\|$. Strictly singular operators share many of the properties of compact operators. However, they form an operator ideal that is strictly larger than the ideal of compact operators. It was thus not clear whether $\mathfrak{X}_{\mathrm{GM}}$ was already a solution of the scalar-plus-compact problem.

Gowers was able to construct a strictly singular, noncompact operator on a subspace of $\mathfrak{X}_{\mathrm{GM}}$ [19]. Thus, $\mathfrak{X}_{\mathrm{GM}}$ could not be a solution of the stronger version of the scalarplus-compact problem asking for all operators from a subspace to the whole space to be compact perturbations of scalar multiples of the inclusion map. Later, George Androulakis and Thomas Schlumprecht [1] showed that strictly singular, non-compact operators exist on the whole space $\mathfrak{X}_{\mathrm{GM}}$. A similar result was shown by Ioannis Gasparis [17] for ArgyrosDeliyanni space (the first example of an asymptotic $\ell_{1} \mathrm{HI}$ space). For these reasons, it became widely accepted that the standard HI constructions would not solve the scalar-pluscompact problem and that a solution was some way away. This is why the landmark result of Argyros and Haydon came as such a surprise not just to the Banach space community but also to the authors. Indeed, Argyros and Haydon did not set out to solve the problem. They were trying to exhibit a hereditarily indecomposable predual of $\ell_{1}$ as an extreme example of the phenomenon that the dual of an HI space need not be HI. They followed the classical ingredients for HI constructions: rapidly increasing sequences of $\ell_{1}$-averages and special functionals using Maurey-Rosenthal coding. However, they did not follow the classical method of constructing HI spaces, which begins with the space of finite sequences and continues with the construction of a suitable exotic norm. Instead, they followed the method of Jean Bourgain and Freddy Delbaen [11] for constructing classes of $\mathscr{L}_{\infty}$-spaces. These are spaces that locally, i.e. at the level of finite-dimensional subspaces, look just like spaces of continuous functions on a compact space and have a priori nothing to do with HI spaces. The Bourgain-Delbaen method is very different from classical HI constructions in that they begin with a very familiar norm, the norm of the space $\ell_{\infty}$ of bounded sequences, and construct their space using carefully chosen exotic vectors in $\ell_{\infty}$. In the delicate construction of Argyros and Haydon, the classical HI ingredients are "woven into" the Bourgain-Delbaen method. Serendipitously, this extra $\mathscr{L}_{\infty}$-structure was exactly what was needed to show that strictly singular operators are compact. In order to explain this remarkable construction, we will have to delve into the constructions of HI spaces and their predecessors: Schlumprecht's space and Tsirelson space. We also need to take a close look at Bourgain-Delbaen spaces and the analysis of their subspace structure by Haydon some 20 years later.

\section{Tsirelson space, Schlumprecht's space and $\mathrm{HI}$ constructions}

Many constructions of Banach spaces, and in particular all the ones described in this section, begin with the space $c_{00}$ of eventually zero scalar sequences. For an element $x=\left(x_{i}\right)$ of $\mathrm{c}_{00}$, we let $\operatorname{supp} x=\left\{i \in \mathbb{N}: x_{i} \neq 0\right\}$ denote the support of $x$. For subsets $A$ and $B$ of $\mathbb{N}$, we write $A<B$ if $a<b$ for all $a \in A$ and $b \in B$; and for $x, y \in \mathrm{c}_{00}$, we write $x<y$ if $\operatorname{supp} x<\operatorname{supp} y$, i.e. if the nonzero coordinates of $x$ 'come before' the nonzero coordinates of $y$. Similarly, we write $n<$ $x$ if $\{n\}<\operatorname{supp} x$, etc.

A norm on $\mathrm{c}_{00}$ is defined by means of a norming set, which is itself a subset of $\mathrm{c}_{00}$. The action of an element $f=\left(f_{i}\right) \in \mathrm{c}_{00}$ on $x=\left(x_{i}\right) \in \mathrm{c}_{00}$ is defined as $\langle x, f\rangle=\sum_{i} x_{i} f_{i}$. Suppose that $W$ is a subset of $\mathrm{c}_{00}$ containing the unit vector basis $\left(e_{n}\right)$ (where $e_{n}$ is the sequence whose $n^{\text {th }}$ coordinate is 1 and all other coordinates are zero) and that $W$ is contained in the unit ball of $\ell_{\infty}$. Then, the expression

$$
\|x\|=\sup \{|\langle x, f\rangle|: f \in W\}
$$


defines a norm on $\mathrm{c}_{00}$ satisfying $\|x\|_{\infty} \leqslant\|x\| \leqslant\|x\|_{1}$, where $\|\cdot\|_{\infty}$ and $\|\cdot\|_{1}$ denote the $\ell_{\infty}$-norm and $\ell_{1}$-norm respectively. The completion $X$ of $\left(c_{00},\|\cdot\|\right)$ is then a Banach space whose structure is determined by the choice of $W$. When considering the subspace structure of $X$, standard perturbation arguments show that it is sufficient to consider only a special type of subspace called a block subspace, which is one generated by a sequence of successive vectors $x_{1}<x_{2}<x_{3}<\ldots$ in $\mathrm{c}_{00}$.

The first space we describe is the famous example of Boris Tsirelson [30]: a Banach space without a subspace isomorphic to one of the classical sequence spaces $\mathrm{c}_{0}$ or $\ell_{p}, 1 \leqslant p<\infty$, which solved a longstanding open problem going back to $\mathrm{Ba}-$ nach's book [9]. It is customary to use the term Tsirelson space for the dual space $T$ of Tsirelson's original construction, which also solves the same problem. The description of $T$ by Tadek Figiel and Bill Johnson [15] is an example of the general process described above for constructing Banach spaces. The norming set $W$ is defined inductively: it is the smallest subset of $\mathrm{c}_{00}$ that contains $\pm e_{n}$ for all $n$ and is closed under the following operation. If $f_{1}<f_{2}<\cdots<f_{n}$ are in $W$ and $n<f_{1}$ then $\frac{1}{2}\left(f_{1}+f_{2}+\cdots+f_{n}\right)$ is also in $W$. Tsirelson space $T$ is then defined to be the completion of $\left(\mathrm{c}_{00},\|\cdot\|\right)$, where the norm $\|\cdot\|$ is defined as in (1) above. Note that without the admissibility condition $n<f_{1}$ or without the factor $\frac{1}{2}$, the space $T$ would be isomorphic to $\ell_{1}$.

The proof that Tsirelson space contains no copy of any of the classical sequence spaces is surprisingly simple. First, it follows easily from the definition of the norming set $W$ that $T$ is an asymptotic $\ell_{1}$ space, which roughly means that for any $n \in \mathbb{N}$, any $n$ vectors 'deep enough' in the space behave like the unit vector basis of $\ell_{1}^{n}$. More precisely, if $n<x_{1}<\cdots<x_{n}$ and $\left\|x_{i}\right\|=1$ for all $i$ then the norm of $\sum_{i=1}^{n} a_{i} x_{i}$ in $T$ is the $\ell_{1}$ norm $\sum_{i=1}^{n}\left|a_{i}\right|$ (up to a factor 2) for arbitrary scalars $a_{i}$. This immediately rules out the possibility of a subspace of $T$ being isomorphic to $\ell_{p}$ for $1<p<\infty$ or to $\mathrm{c}_{0}$.

Proving that $T$ contains no copy of $\ell_{1}$ requires a bit more effort. This should not come as a surprise since, as we saw above, $T$ is, in an asymptotic sense, close to $\ell_{1}$. The proof is indirect: one demonstrates a hereditary property of $T$ (i.e. one that passes to all subspaces) that $\ell_{1}$ lacks. This property is distortability: an important property in its own right and one that is intertwined with the development of HI spaces. We say that a Banach space $X$ with norm $\|\cdot\|$ is distortable if there exist $\lambda>1$ and an equivalent norm $\|\cdot|\|| \mid$ on $X$ such that for every (infinite-dimensional) subspace $Y$ of $X$, there are vectors $y, z \in Y$ with $\|y\|=\|z\|=1$ and $\|y\| /\|z\| \|>$. In other words, $\|\cdot\|$ and $\|\cdot\| \cdot \|$ are not $\lambda$-equivalent, not even after passing to a subspace. In this case, we say that $X$ is $\lambda$-distortable. A Banach space is arbitrarily distortable if it is $\lambda$-distortable for all $\lambda>1$. An old result of Robert James [24] says that $\ell_{1}$ and $\mathrm{c}_{0}$ are not distortable, which means that a Banach space containing an isomorphic copy of one of these sequence spaces also contains almost isometric copies. Hence, a distortable space cannot contain a subspace isomorphic to $\ell_{1}$ or $\mathrm{c}_{0}$.

The proof that $T$ is distortable features an ingredient that is crucial in all HI constructions: rapidly increasing sequences of $\ell_{1}$-averages. We say that $x \in \mathrm{c}_{00}$ is an $\ell_{1}^{n}$-vector of constant $C$ if there is a decomposition $x=x_{1}+x_{2}+\cdots+x_{n}$ of $x$ such that $x_{1}<x_{2}<\cdots<x_{n}$ and $\left\|x_{i}\right\| \leqslant \frac{C}{n}\|x\|$ for all $i$. If, in addition, we have $\|x\|=1$ then $x$ is called an $\ell_{1}^{n}$-average of constant $C$ or an $\ell_{1}$-average of constant $C$ and length $n$. (Here $\|\cdot\|$ is the norm of $T$ but the definitions make sense for any norm on $\mathrm{c}_{00}$.) Note that, by the triangle inequality, $C$ is necessarily at least 1 . We say that a (finite or infinite) sequence $x_{1}<x_{2}<\ldots$ is a rapidly increasing sequence (RIS) if $x_{i}$ is an $\ell_{1}^{n_{i}}$-average of constant very close to 1 and $n_{1}<n_{2}<\ldots$ is a very fastgrowing sequence of integers. (These conditions are of course made precise.) If $x=x_{1}+x_{2}+\cdots+x_{n}$, where $x_{1}<x_{2}<$ $\cdots<x_{n}$ is an RIS of length $n$, then we call $x$ an RIS vector (of length $n$ ) and $x /\|x\|$ a normalised RIS vector (of length $n)$.

The earlier observation that $T$ is asymptotic $\ell_{1}$ shows that $T$, and indeed every block subspace of $T$, contains $\ell_{1}^{n}$ averages of constant 2 for any $n$. James' argument showing that $\ell_{1}$ is not distortable can be generalised to prove the following: if a space contains, for some $C>1$ and for all $n, \ell_{1}^{n}$ averages of constant $C$ then it also contains, for all $C>1$ and for all $n, \ell_{1}^{n}$-averages of constant $C$. In particular, this holds in Tsirelson space and in all its block subspaces. In turn, this also implies that every block subspace of $T$ contains RIS vectors of length $n$ for all $n$. Let us now define a new norm $\|\cdot\| \cdot \|$ on $T$ as follows. Fix some large $m \in \mathbb{N}$ and for $x \in T$, define

$$
\|x\|=\sup \sum_{i=1}^{m}\left\|y_{i}\right\|,
$$

where the supremum is over all decompositions $x=y_{1}+\cdots+$ $y_{m}$ of $x$ with $y_{1}<\cdots<y_{m}$. This is clearly $m$-equivalent to the original norm. By definition, an $\ell_{1}^{n}$-average $x$ of constant $C$ comes with a specific decomposition $x=x_{1}+\cdots+x_{n}$, where $x_{1}<\cdots<x_{n}$ and $\left\|x_{i}\right\| \leqslant \frac{C}{n}$ for all $i$. These $x_{i}$ add up in an $\ell_{1}$-fashion in the sense that $\sum\left\|x_{i}\right\|$ is at most $C$. It turns out that the same is true for any decomposition of $x$; more precisely, if $x=y_{1}+\cdots+y_{m}$, where $y_{1}<\cdots<y_{m}$, then $\sum\left\|y_{i}\right\|$ is at most $C\left(1+\frac{m}{n}\right)$. Thus, if $x$ is an $\ell_{1}^{n}$-average of constant $C$ with $C$ very close to 1 and with $n$ much larger than $m$ then $\|x\| \approx$ $1=\|x\|$. RIS vectors, on the other hand, behave differently. Let $z=z_{1}+\cdots+z_{m}$ be an RIS vector of length $m$, where $z_{1}<\cdots<z_{m}$ and $z_{i}$ is an $\ell_{1}^{n_{i}}$-average of constant close to 1 and with $n_{1}<\cdots<n_{m}$ growing rapidly as in the definition. Then, $\|z\| \geqslant \geqslant \sum_{i=1}^{m}\left\|z_{i}\right\|=m$. Since $\left\|z_{i}\right\|=1$ for all $i$, there exist $f_{1}<\cdots<f_{m}$ in $W$ such that $\left\langle z_{i}, f_{i}\right\rangle \approx 1$ for all $i$. Assuming, for argument's sake, that $m<f_{1}$, we have $f=\frac{1}{2}\left(f_{1}+\cdots+\right.$ $\left.f_{m}\right) \in W$ and hence $\|z\| \geqslant\langle z, f\rangle \approx m / 2$. The key combinatorial lemma for $T$ shows that this lower bound is essentially the best possible, i.e. that $\|z\| \approx m / 2$. The proof makes important use of the rapid growth of $n_{1}<n_{2}<\cdots<n_{m}$. Thus, every block subspace of $T$ contains a normalised vector whose $\|\cdot\| \cdot \|-$ norm is at least approximately 2 . This demonstrates that $T$ is $\lambda$-distortable for any $\lambda<2$.

We remark that if we replace the factor $\frac{1}{2}$ in the definition of the norming set $W$ of $T$ by an arbitrary $\theta \in(0,1)$, we obtain a space that is $\lambda$-distortable for all $\lambda<\theta^{-1}$. Thus, for all $\lambda>1$, there exists a $\lambda$-distortable Banach space. It is a famous open problem whether $T$ is arbitrarily distortable. For some while, it was not known if such a space exists at all. A remarkable space constructed by Schlumprecht [29] provided the first example, as well as a key step toward subsequent constructions of HI spaces.

Schlumprecht's space $S$ is defined in a similar way to Tsirelson space. We introduce the function $\varphi(x)=\log _{2}(1+x)$ 
defined on $[1, \infty)$ and take our norming set to be the smallest subset $W$ of $\mathrm{c}_{00}$ containing $\pm e_{n}$ for all $n$ and closed under the following operation: for any $n \in \mathbb{N}$, if $f_{1}<f_{2}<\cdots<f_{n}$ belong to $W$ then so does $f=\varphi(n)^{-1}\left(f_{1}+\cdots+f_{n}\right)$. So, we no longer require the $f_{i}$ to be 'far out' in $\mathrm{c}_{00}$; instead, there is a higher price to be paid for a large value of $n$, namely the factor $\varphi(n)^{-1}$ replacing the constant factor $1 / 2$ of $T$. The space $S$ is the completion of $\left(\mathrm{c}_{00},\|\cdot\|\right)$, where the norm $\|\cdot\|$ is defined as in (1) above.

The proof that $S$ is arbitrarily distortable is similar to the argument used in $T$ : the different behaviour of $\ell_{1}$-averages and RIS vectors is key. We fix a large $m \in \mathbb{N}$ and define an equivalent norm $\|\cdot \mid\|$ for $S$ by (2). Again using James' argument, it is not hard to show that every block subspace of $S$ contains $\ell_{1}^{n}$-averages of constant $C$ for all $C>1$ and for all $n$, and hence RIS vectors of all lengths. Again, if $x$ is an $\ell_{1}^{n}$ average of constant $C$ with $C$ very close to 1 and with $n$ much larger than $m$ then $\|x\| \approx 1$. On the other hand, RIS vectors behave very differently. Let $z=z_{1}+\cdots+z_{m}$ be an RIS vector of length $m$, where $z_{1}<\cdots<z_{m}$ and $z_{i}$ is an $\ell_{1}^{n_{i}}$-average of constant close to 1 and with $n_{1}<\cdots<n_{m}$ growing rapidly as in the definition. Then, $\|z\| \geqslant \sum_{i=1}^{m}\left\|z_{i}\right\|=m$. Since $\left\|z_{i}\right\|=1$ for all $i$, there exist $f_{1}<\cdots<f_{m}$ in $W$ such that $\left\langle z_{i}, f_{i}\right\rangle \approx 1$ for all $i$. Then, $f=\varphi(m)^{-1}\left(f_{1}+\cdots+f_{m}\right) \in W$ and hence $\|z\| \geqslant\langle z, f\rangle \approx m / \varphi(m)$. The key combinatorial lemma, as in $T$ but this time much harder to prove, says that this lower bound is essentially the best possible, i.e. that $\|z\| \approx m / \varphi(m)$. Thus, every block subspace of $T$ contains a normalised vector whose $\|\cdot\| \cdot \|$-norm is at least approximately $\varphi(m)$. This shows that $S$ is arbitrarily distortable.

The space $\mathfrak{X}_{G M}$ of Gowers and Maurey [21] was the first example of an hereditarily indecomposable Banach space and was a development of Schlumprecht's space. In addition to rapidly increasing sequences of $\ell_{1}$-averages, the construction also made use of Maurey-Rosenthal coding. This was introduced by Bernard Maurey and Haskell Rosenthal in their construction of a normalised weakly null sequence without an unconditional subsequence [27]. As with the spaces $T$ and $S$, the Gowers-Maurey space $\mathfrak{X}_{G M}$ is the completion of $\left(\mathrm{c}_{00},\|\cdot\|\right)$, where the norm $\|\cdot\|$ is defined by (1) with a suitably chosen $W \subset \mathrm{c}_{00}$. The norming set $W$ is defined as the smallest subset of $\mathrm{c}_{00}$ containing $\pm e_{n}$ for all $n$ and satisfying certain closure properties. These include the one already used in the construction of $S$ : if $f_{1}<\cdots<f_{n}$ are in $W$ then so is $\varphi(n)^{-1}\left(f_{1}+\cdots+f_{n}\right)$. In addition, a crucial role is played by functionals of the form $\varphi(k)^{-1 / 2}\left(g_{1}+\cdots+g_{k}\right)$ for certain special sequences $g_{1}, \ldots, g_{k}$ of elements of $W$. These have a certain tree-like structure: if $g_{1}, g_{2}, \ldots$ and $h_{1}, h_{2}, \ldots$ are special sequences and $i$ is minimal such that $g_{i} \neq h_{i}$ then, for all $j, l>i$, the functionals $g_{j}$ and $h_{l}$ behave very differently. This is achieved by an injective function $\sigma$ called the MaureyRosenthal coding: $\sigma\left(g_{1}, \ldots, g_{j-1}\right)$ defines a certain parameter of $g_{j}$. These so-called special functionals are in some sense rare and, in particular, their length $k$ must come from some very lacunary subset of $\mathbb{N}$.

It is again fairly straightforward to establish that every block subspace of $\mathfrak{X}_{G M}$ contains $\ell_{1}$-averages of arbitrary length and constant arbitrarily close to 1 , and hence RIS vectors of arbitrary length. The key inequality of $S$ also holds in $\mathfrak{\mathfrak { t }}_{G M}$ with some restriction: if $x$ is an RIS vector in $\mathfrak{X}_{G M}$ of length $m$ then $\|x\| \approx m / \varphi(m)$, provided $x$ is roughly speaking not aligned with a special functional that would force it to have a much bigger norm of at least $m / \sqrt{\varphi(m)}$. In particular, if $m$ is far from the lacunary set of possible lengths of special sequences then $\|x\| \approx m / \varphi(m)$ always holds.

The key properties of $T$ and $S$ come from the different behaviour of $\ell_{1}$-averages and RIS vectors. If we think of an RIS vector built from $\ell_{1}$-averages as having complexity 1 then, in showing that $\mathfrak{X}_{G M}$ is hereditarily indecomposable, we shall need rapidly increasing sequences of complexity 2 . By this, we mean sequences $x_{1}<\cdots<x_{m}$, where each $x_{i}$ is a normalised RIS vector of some length $n_{i}$ and $n_{1}<\cdots<n_{m}$ is a fast-growing sequence. The difference in behaviour comes from the alignment or otherwise of this complexity-2 RIS with a special sequence of functionals.

An easy equivalent definition of $\mathrm{HI}$ goes as follows. A Banach space $X$ is hereditarily indecomposable if and only if, for any two (infinite-dimensional) subspaces $Y$ and $Z$ of $X$, the ratio $\|y+z\| /\|y-z\|, y \in Y$ and $z \in Z$, can get arbitrarily large. Informally, the 'angle' between any two subspaces is zero. Assume then that $Y$ and $Z$ are subspaces of $X$, which can be taken to be block subspaces after perturbation. It is then possible to build a complexity-2 RIS $x_{1}<\cdots<x_{k}$ together with a special sequence $g_{1}<\cdots<g_{k}$ of functionals such that $x_{i} \in Y$ for all odd values of $i, x_{i} \in Z$ for all even values of $i$ and $\left\langle x_{i}, g_{i}\right\rangle \approx 1$. Set $y=x_{1}+x_{3}+\ldots, z=x_{2}+x_{4}+\ldots$ and $g=\varphi(k)^{-1 / 2}\left(g_{1}+\cdots+g_{k}\right)$. Then, $y \in Y, z \in Z$ and $g \in W$ is a special functional. It follows that

$$
\|y+z\| \geqslant\langle y+z, g\rangle=\varphi(k)^{-1 / 2} \sum\left\langle x_{i}, g_{i}\right\rangle \approx k / \sqrt{\varphi(k)} .
$$

On the other hand, $\langle y-z, g\rangle=\varphi(k)^{-1 / 2} \sum(-1)^{i-1}\left\langle x_{i}, g_{i}\right\rangle \approx 0$ because of cancellations. Due to the tree-like structure of special functionals, the same holds for any special functional of length $k$. This is one of the situations when the key inequality of $S$ applies, and we obtain $\|y-z\| \approx k / \varphi(k)$. The ratio $\|y+z\| /\|y-z\| \approx \sqrt{\varphi(k)}$ gets arbitrarily large and so $\mathfrak{X}_{G M}$ is hereditarily indecomposable.

As mentioned earlier, on the space $\mathfrak{X}_{G M}$, every operator is a strictly singular perturbation of a scalar multiple of the identity. This can be seen as follows. Given a bounded linear operator $T$ on $\mathfrak{X}_{G M}$, we first find $\lambda \in \mathbb{R}$ such that $(T-\lambda) x_{i} \rightarrow 0$ for every infinite rapidly increasing sequence $\left(x_{i}\right)$. This is done in two steps. First one shows that $d\left(T x_{i}, \mathbb{R} x_{i}\right) \rightarrow 0$ as $i \rightarrow \infty$. If that were not the case then we could build an RIS vector $x$ of complexity 2 (as in the proof that $\mathfrak{X}_{G M}$ is $\mathrm{HI}$ ) such that $\|T x\| /\|x\|>\|T\|$. It then follows that for some $\lambda \in \mathbb{R}$, we have $(T-\lambda) x_{i} \rightarrow 0$ as $i \rightarrow \infty$. By "merging" two infinite rapidly increasing sequences, it is clear that $\lambda$ does not depend on $\left(x_{i}\right)$. Finally, since every block subspace contains a rapidly increasing sequence, it follows that $T-\lambda$ is strictly singular. It is worth pointing out that for the stronger property of compactness, we would need $(T-\lambda) x_{i} \rightarrow 0$ for every bounded sequence $x_{1}<x_{2}<\ldots$.

Following the first construction of an HI space by Gowers and Maurey, the class of HI spaces has been studied extensively by many people, notably by Argyros and his coauthors. The motivation for this was Gowers' famous dichotomy theorem: for every Banach space $X$, either $X$ is unconditionally saturated (every subspace of $X$ contains a fur- 
ther subspace with an unconditional basis) or $X$ has an $\mathrm{HI}$ subspace. It was therefore of interest to better understand the structure of HI spaces: their duality, quotients and operator algebras. In [2], Argyros showed that if a separable Banach space $Z$ contains isomorphic copies of every separable, reflexive $\mathrm{HI}$ space then $Z$ must, in fact, be universal, i.e. it must contain isomorphic copies of all separable Banach spaces. Thus, HI spaces are in some sense ubiquitous despite being so difficult to come by. Extending earlier work of Argyros and Vaggelis Felouzis [3], Argyros and Andreas Tolias [7] showed that every separable Banach space either contains $\ell_{1}$ or is the quotient of an HI space, which implies that the class of HI spaces and their duals is very rich. A remarkable result of Argyros and Tolias [6] shows that there is a dual pair of Banach spaces: one is HI and the other is unconditionally saturated, i.e. they are on opposite sides of Gowers' Dichotomy. All these examples are Tsirelson-like constructions: they begin with $\mathrm{c}_{00}$ and a norming set $W$ closed under certain operations that are defined by a sequence $\left(\theta_{j}, \mathcal{A}_{j}\right)$, where $\theta_{j} \in(0,1)$ and $\mathcal{A}_{j}$ is a family of finite subsets of $\mathbb{N}$ for each $j \in \mathbb{N}$. The norming set $W$ is defined to be the smallest subset of $\mathrm{c}_{00}$ containing $\pm e_{n}$ for all $n$ such that if $f_{1}<\cdots<f_{n}$ are in $W$ and $\left\{\min \operatorname{supp} f_{i}: 1 \leqslant i \leqslant n\right\} \in \mathcal{A}_{j}$ then $\theta_{j}\left(f_{1}+\cdots+f_{n}\right) \in W$. Note that if $\theta_{j}=\frac{1}{2}$ and $\mathcal{A}_{j}=\{A \subset \mathbb{N}:|A| \leqslant \min A\}$ for all $j$ then we obtain Tsirelson space $T$. If $\theta_{j}=\log _{2}(1+j)^{-1}$ and $\mathcal{A}_{j}=\{A \subset \mathbb{N}:|A| \leqslant j\}$ for all $j$ then we recover Schlumprecht's space $S$. It turns out that the classical sequence spaces $\ell_{p}$ can also be obtained by a suitable choice of $\left(\theta_{j}, \mathcal{A}_{j}\right)$. This was shown by Steven Bellenot in [10]. There is an alternative Tsirelson-like construction, which yields a norm equivalent to the $\ell_{p}$-norm and which has a place in the story of the solution to the scalar-plus-compact problem. Fix real numbers $a, b \in(0,1)$ with $a+b>1$ and let $W$ be the smallest subset of $\mathrm{c}_{00}$ containing $\pm e_{n}$ for all $n$, such that if $f<g$ are in $W$ then so is $a f+b g$. Let us denote by $U_{a, b}$ the completion of $\left(c_{00},\|\cdot\|\right)$, where $\|\cdot\|$ is defined by (1) for this choice of $W$. It was shown by Haydon in his work [22] on Bourgain-Delbaen spaces that $U_{a, b}$ is isomorphic to $\ell_{p}$, where $p$ is determined by the equations $1 / p+1 / p^{\prime}=1=$ $a^{p^{\prime}}+b^{p^{\prime}}$.

\section{The Bourgain-Delbaen construction}

We now turn to the other line of research that paved the way to the solution of the scalar-plus-compact problem. Recall that a Banach space $X$ is a $\mathscr{L}_{\infty}$-space if for some $\lambda \geqslant 1$ and for every finite-dimensional subspace $E$ of $X$, there is a finite-dimensional subspace $F$ of $X$ such that $E \subset F$ and the Banach-Mazur distance $d\left(F, \ell_{\infty}^{n}\right) \leqslant \lambda$, where $n=\operatorname{dim} F$. Thus, locally, $\mathscr{L}_{\infty}$-spaces behave like $C(K)$ spaces, i.e. spaces of continuous functions on compact Hausdorff spaces. For this reason, it was believed that, in some sense, a $\mathscr{L}_{\infty}$-space cannot be too far from a $C(K)$ space. It therefore came as a surprise when Bourgain and Delbaen [11] constructed new examples that showed a great diversity of $\mathscr{L}_{\infty}$-spaces and settled several open problems in the theory of Banach spaces. In particular, they constructed separable $\mathscr{L}_{\infty}$-spaces not containing $\mathrm{c}_{0}$ whose dual is $\ell_{1}$. More precisely, for given real numbers $a, b$ satisfying $0<b<1 / 2<a<1$ and $a+b>1$, they constructed a separable $\mathscr{L}_{\infty}$-space $X_{a, b}$ that is somewhat reflexive (every subspace contains a further subspace that is reflexive) and whose dual is isomorphic to $\ell_{1}$. The proof that $X_{a, b}$ is somewhat reflexive uses norm estimates involving the space $U_{a, b}$ defined above. Much later, Haydon carried out a careful analysis of the subspace structure of $X_{a, b}$. In [22], he proved that every block subspace of $X_{a, b}$ contains a subspace isomorphic to $U_{a, b}$ and that $U_{a, b}$ is isomorphic to some $\ell_{p}$ space.

All constructions of HI spaces before Argyros and Haydon begin with $\mathrm{c}_{00}$ and equip it with some exotic norm. The Bourgain-Delbaen construction is very different: they construct their space inside $\ell_{\infty}$ by choosing exotic vectors. We now describe this in a bit more detail, following [4]. The vectors constructed inside $\ell_{\infty}$ will be the biorthogonal functionals of a basis of $\ell_{1}$. Denoting by $X$ the subspace of $\ell_{\infty}$ generated by these vectors, $\ell_{1}$ embeds into $X^{*}$ (and will sometimes be the whole of $X^{*}$ ). For this reason, we think of elements of $\ell_{1}$ as functionals. Accordingly, we denote by $\left(e_{n}^{*}\right)$ the unit vector basis of $\ell_{1}$, whereas we use the notation $\left(e_{n}\right)$ for the same vectors when inside $\ell_{\infty}$. From now on, we shall also think of elements of $\ell_{\infty}$ as functions $x: \mathbb{N} \rightarrow \mathbb{R}$ and write $x(\gamma)$ instead of $x_{\gamma}$ for $\gamma \in \mathbb{N}$. The support supp $x$ of $x$ now has the usual meaning for functions: $\operatorname{supp} x=\{\gamma \in \mathbb{N}: x(\gamma) \neq 0\}$.

A basis $\left(d_{n}^{*}\right)$ of $\ell_{1}$ is called triangular if $d_{n}^{*}=\sum_{j=1}^{n} a_{n, j} e_{j}^{*}$ with $a_{n, n} \neq 0$. We shall always take $a_{n, n}=1$. In that case, the biorthogonal vectors $\left(d_{n}\right)$ in $\ell_{\infty}$ form a basis of their closed linear span $X$. Moreover, for each $n \in \mathbb{N}$, the space $\operatorname{span}\left\{d_{1}, \ldots, d_{n}\right\}$ is isomorphic to $\ell_{\infty}^{n}$ via the map sending $x$ to its restriction to $\{1, \ldots, n\}$. To put it in another way, each $u \in \ell_{\infty}^{n}$ has a unique extension $x$ into $\operatorname{span}\left\{d_{1}, \ldots, d_{n}\right\}$ with $x(\gamma)=u(\gamma)$ for all $\gamma \leqslant n$. This property will be crucial for the scalar-plus-compact property of the Argyros-Haydon space. The isomorphism constant between $\operatorname{span}\left\{d_{1}, \ldots, d_{n}\right\}$ and $\ell_{\infty}^{n}$ turns out to be bounded by the basis constant of $\left(d_{i}^{*}\right)$, which implies that $X$ is a $\mathscr{L}_{\infty}$-space. The basis $\left(d_{n}^{*}\right)$ is constructed recursively: at each step, an element $c_{n}^{*} \in \operatorname{span}\left\{e_{i}^{*}: i<n\right\}$ is chosen and $d_{n}^{*}$ is defined to be $d_{n}^{*}=e_{n}^{*}-c_{n}^{*}$. (The recursion is actually done in batches and, at each step, several functionals are chosen at once.) Of course, the clever part of the Bourgain-Delbaen construction lies in the choice of the vectors $\left(c_{i}^{*}\right)$. Since the norm on the space $X$ is simply the $\ell_{\infty}$ norm, one needs estimates on how the evaluation functionals $e_{n}^{*}$ act on certain vectors. Observing that $e_{n}^{*}=d_{n}^{*}+c_{n}^{*}$ and using induction, one can express these evaluation functionals as sums of successive functionals, which is called the evaluation analysis by Argyros and Haydon. In the Bourgain-Delbaen spaces $X_{a, b}$, the evaluation analysis features the functionals that define the spaces $U_{a, b}$. This makes the way in which the Tsirelson-like construction of $U_{a, b}$ is "woven" into the construction of $X_{a, b}$ more transparent.

\section{The Argyros-Haydon space}

We now finally turn our attention to the space $\mathfrak{X}_{A H}$ of Argyros and Haydon. As mentioned already, their initial aim was to construct a separable $\mathscr{L}_{\infty}$-space that is hereditarily indecomposable. Their idea was that since it was possible to recursively build into the dual of the Bourgain-Delbaen space $X_{a, b}$ the Tsirelson-like norming set of $U_{a, b}$, the same can perhaps be done with much more sophisticated Tsirelson-like 
constructions. Indeed, this was already done by Haydon for Tsirelson space $T$ in [23]. The remarkable feat of Argyros and Haydon was to take this much further and build a BourgainDelbaen space modelled on a Tsirelson-like HI space. The construction begins with fixing two very fast-growing sequences $\left(m_{i}\right)$ and $\left(n_{i}\right)$ of positive integers. For $j \in \mathbb{N}$, let $\theta_{j}=m_{j}^{-1}$ and $\mathcal{A}_{j}=\left\{A \subset \mathbb{N}:|A| \leqslant 4 n_{j}\right\}$. The sequence $\left(\theta_{j}, \mathcal{A}_{j}\right)$ then defines a Tsirelson-like space $T\left[\left(\theta_{j}, \mathcal{A}_{j}\right)\right]$ and this forms the backbone of $\mathfrak{X}_{A H}$, together with a MaureyRosenthal coding used in the construction of $\mathfrak{X}_{G M}$. As for the original Bourgain-Delbaen spaces, a triangular basis $\left(d_{n}^{*}\right)$ of $\ell_{1}$ is constructed recursively in batches. This leads to a partitioning of $\mathbb{N}$ into intervals $\Delta_{1}<\Delta_{2}<\ldots$, where by an interval we mean a set of the form $\{m, m+1, \ldots, n\}$ for some $m \leqslant n$ in $\mathbb{N}$. We begin with setting $\Delta_{1}=\{1\}$ and $d_{1}^{*}=e_{1}^{*}$. The construction then proceeds in stages. At the $n^{\text {th }}$ stage, intervals $\Delta_{1}<\cdots<\Delta_{n}$ and triangular basis vectors $d_{\gamma}^{*}$, $\gamma \in \Gamma_{n}=\bigcup_{i=1}^{n} \Delta_{i}$ for $\ell_{1}\left(\Gamma_{n}\right)$ are assumed to have been constructed. We then fix a certain, carefully chosen finite subset of $\ell_{1}\left(\Gamma_{n}\right)$ enumerated as $b_{\gamma}^{*}, \gamma \in \Delta_{n+1}$, which then defines the next interval $\Delta_{n+1}$. The $n^{\text {th }}$ stage of the construction is completed by setting $d_{\gamma}^{*}=e_{\gamma}^{*}-b_{\gamma}^{*}$ for each $\gamma \in \Delta_{n+1}$. This process then produces a basis $\left(d_{\gamma}^{*}\right)_{\gamma \in \mathbb{N}}$ of $\ell_{1}$. As before, we now denote by $\left(d_{n}\right)$ the biorthogonal sequence in $\ell_{\infty}$. The space $\mathfrak{X}_{A H}$ is defined to be the closed linear span of $\left(d_{n}\right)$ in $\ell_{\infty}$. It follows that $\mathfrak{X}_{A H}$ is a $\mathscr{L}_{\infty}$-space with basis $\left(d_{n}\right)$.

The key to the construction is of course the choice of the functionals $b_{\gamma}^{*}$ in the recursive construction of the basis of $\ell_{1}$. This is done in such a way that, for $\gamma \in \mathbb{N}$, the evaluation analysis of $e_{\gamma}^{*}$ has the following form:

$$
e_{\gamma}^{*}=\sum_{r=1}^{a} d_{\xi_{r}}^{*}+m_{j}^{-1} \sum_{r=1}^{a} x_{r}^{*},
$$

where $1 \leqslant \xi_{1}<\xi_{2}<\cdots<\xi_{a}=\gamma$ and the $x_{i}^{*}$ are functionals so that the second term $m_{j}^{-1} \sum_{r=1}^{a} x_{r}^{*}$ is one of the norming functionals of the Tsirelson-like space $T\left[\left(\theta_{j}, \mathcal{A}_{j}\right)\right]$. The factor $m_{j}^{-1}$ here is called the weight of $\gamma$, denoted $w(\gamma)$. The recursive construction of the basis $\left(d_{\gamma}^{*}\right)$ of $\ell_{1}$ is carried out in a way that ensures that there is an abundance of possible evaluation analyses produced by those $\gamma$ that have even weight (meaning that $j$ is even). On the other hand, there is a restriction on evaluation functionals of odd weight governed by a certain injective function $\sigma: \mathbb{N} \rightarrow \mathbb{N}$. This coding function is constructed alongside the triangular basis in the recursive process at the start. Now, if $\gamma \in \mathbb{N}$ has odd weight, say $w(\gamma)=m_{2 j-1}^{-1}$, then $e_{\gamma}^{*}$ has evaluation analysis

$$
e_{\gamma}^{*}=\sum_{r=1}^{a} d_{\xi_{r}}^{*}+m_{2 j-1}^{-1} \sum_{r=1}^{a} y_{r}^{*},
$$

where each $y_{r}^{*}$ is some projection of an evaluation functional $e_{\eta_{r}}^{*}$, with $e_{\eta_{r}}^{*}$ having even weight $w\left(\eta_{r}\right)=m_{4 \sigma\left(\xi_{r-1}\right)}^{-1}$ for $1<r \leqslant$ $a$. Clearly, these evaluation functionals of odd weight correspond to the special functionals in Gowers-Maurey space. Because of the injectivity of $\sigma$, these evaluation functionals also have a tree-like structure.

Let us now turn to the properties of $\mathfrak{X}_{A H}$. As in any Tsirelson-like space, from the abundance of functionals of even weight, one can deduce that, for every $C>1$, every block subspace of $\mathfrak{X}_{A H}$ contains arbitrarily long $\ell_{1}$-averages of constant $C$. Recall that an $\ell_{1}^{n}$-average of constant $C$ comes with a decomposition as a sum of $n$ vectors that add in an $\ell_{1}$ fashion up to a factor $C$, and that the same holds for any decomposition into $m$ successive vectors as long as $m$ is much smaller than $n$. The analogous result in $\mathfrak{X}_{A H}$ has a similar proof and is as follows. If $x$ is an $\ell_{1}^{n_{j}}$-average of constant $C$ and $\gamma$ is of weight $m_{i}^{-1}$ with $i<j$ then $|x(\gamma)| \leqslant 2 C m_{i}^{-1}$. The next step is to define rapidly increasing sequences. Rather than defining this as a sequence of $\ell_{1}$-averages, we instead use the property of $\ell_{1}$-averages just observed. So, a (finite or infinite) sequence $x_{1}<x_{2}<\ldots$ is a rapidly increasing sequence of constant $C$ (or $C$-RIS) if:

(i) $\left\|x_{k}\right\| \leqslant C$ for all $k$

and there exists a fast-growing sequence $j_{1}<j_{2}<\ldots$ such that, for all $k$,

(ii) $\left|x_{k}(\gamma)\right| \leqslant C m_{i}^{-1}$ whenever $w(\gamma)=m_{i}^{-1}$ and $i<j_{k}$. It follows from above that, for every $C>2$, every block subspace of $\mathfrak{X}_{A H}$ contains a $C$-RIS.

Recall that the key inequality in Schlumprecht's space says that an RIS vector of length $m$ has norm at most approximately $m / \varphi(m)$. There is an analogue in $\mathfrak{X}_{A H}$ with a similar proof and it is, in turn, a special case of what Argyros and Haydon call the basic inequality. It says that if $\left(x_{k}\right)_{k=1}^{n_{j_{0}}}$ is a $C$-RIS then

$$
\left\|\sum_{k=1}^{n_{j_{0}}} x_{k}\right\| \leqslant 10 C \frac{n_{j_{0}}}{m_{j_{0}}} .
$$

(Note that in $S$ one has $n_{j_{0}}=j_{0}$ and $m_{j_{0}}=\varphi\left(j_{0}\right)$.) Using terminology introduced earlier, the RIS above might be called a complexity-1 RIS. For a complexity-2 RIS, which can be built as in $\mathfrak{X}_{G M}$, there is a better norm estimate. Argyros and Haydon introduce the notion of an exact pair, examples of which include normalised RIS vectors. One can then recursively build sequences $\left(x_{k}\right)_{k=1}^{n_{2 j_{0}-1}}$ of exact pairs, called dependent sequences, together with functionals that will end up being the evaluation analysis of some $e_{\gamma}^{*}$ of odd weight $m_{2 j_{0}-1}^{-1}$. This is analogous to building complexity-2 RIS vectors and special functionals in the proof that $\mathfrak{X}_{G M}$ is hereditarily indecomposable.

There are, in fact, two types of exact pairs (and hence dependent sequences): 0-pairs and 1-pairs. (This is not the exact terminology of [4].) As the name suggests, an exact pair is not just a vector $x$ but a pair $(x, \eta)$, where $\eta \in \mathbb{N}$ and $x(\eta)$ is 0 or 1 . When the $x_{k}$ are type-1 exact pairs taken from given block subspaces $Y$ and $Z$ by alternating between them, the resulting dependent sequence will satisfy the norm estimate

$$
\left\|\sum_{k=1}^{n_{2 j_{0}-1}}(-1)^{k} x_{k}\right\| \leqslant 40 C \frac{n_{j_{0}}}{m_{j_{0}}^{2}},
$$

which is an improvement on (3). This roughly follows from the fact that evaluating the above sum at $\gamma$ would give something small and so, by the tree-like structure of odd-weight evaluation functionals, every other $e_{\gamma^{\prime}}^{*}$ of weight $m_{2 j_{0}-1}^{-1}$ is small on the sum. On the other hand, evaluation at $\gamma$ gives

$$
\left\|\sum_{k=1}^{n_{2 j_{0}-1}} x_{k}\right\| \geqslant \frac{n_{j_{0}}}{m_{j_{0}}} .
$$

This already implies that $\mathfrak{X}_{A H}$ is hereditarily indecomposable.

We now turn to operators. Assume that $T$ is a bounded linear operator on $\mathfrak{X}_{A H}$. Assume that $\left(x_{i}\right)$ is an infinite $C$-RIS 
such that $d\left(T x_{i}, \mathbb{R} x_{i}\right) \nrightarrow 0$. Using Hahn-Banach, one can then find $\delta>0$ and build exact 0 -pairs $(z, \eta)$ with $T z(\eta)>\delta$. One can then recursively construct a dependent sequence $\left(z_{k}\right)_{k=1}^{n_{2 j_{0}-1}}$ of 0-pairs that forms an RIS, and for which one gets the improved estimate

$$
\left\|\sum_{k=1}^{n_{2 j_{0}-1}} z_{k}\right\| \leqslant C^{\prime} \frac{n_{j_{0}}}{m_{j_{0}}^{2}}
$$

where $C^{\prime}$ is a constant depending on $C$. On the other hand, the recursive construction produces an evaluation functional $e_{\gamma}^{*}$ of weight $m_{2 j_{0}-1}^{-1}$ for which

$$
\sum_{k=1}^{n_{2 j_{0}-1}} T z_{k}(\gamma) \geqslant \delta^{\prime} \frac{n_{j_{0}}}{m_{j_{0}}}
$$

where $\delta^{\prime}$ depends only on $\delta$. Putting $z=\sum_{k=1}^{n_{2 j_{0}-1}} z_{k}$, we obtain $\|T z\| /\|z\| \geqslant \delta^{\prime} m_{j_{0}} / C^{\prime}$, which is a contradiction for large $j_{0}$. It follows that $d\left(T x_{i}, \mathbb{R} x_{i}\right) \rightarrow 0$. It is not difficult to deduce that there is a $\lambda \in \mathbb{R}$ such that $(T-\lambda) x_{i} \rightarrow 0$ for every RIS $\left(x_{i}\right)$. This already implies that $T-\lambda$ is strictly singular.

We now come to the most important property of $\mathfrak{X}_{A H}$ and show that the above operator $T-\lambda$ is in fact compact. At this point the "special functionals", i.e. evaluations of odd weight, play no role whatsoever; instead, the $\mathscr{L}_{\infty}$-structure becomes important. Suppose we are given $x \in \mathfrak{X}_{A H}$, which we write as $x=\sum_{\gamma \in \mathbb{N}} a_{\gamma} d_{\gamma}$ in terms of the basis $\left(d_{\gamma}\right)$. Assume $x$ has finite support and $n$ is minimal so that $\{\gamma \in \mathbb{N}$ : $\left.a_{\gamma} \neq 0\right\} \subset \Gamma_{n}=\bigcup_{i=1}^{n} \Delta_{i}$. Call this $n$ the maximum range of $x$, denoted max ran $x$. Let $u \in \ell_{\infty}\left(\Gamma_{n}\right)$ be the restriction of $x$ to $\Gamma_{n}$. Recall that $x$ is the unique element of $\operatorname{span}\left\{d_{\gamma}: \gamma \in \Gamma_{n}\right\}$ that extends $u$, i.e. for which $x(\gamma)=u(\gamma)$ for all $\gamma \in \Gamma_{n}$ and, moreover, the map $u \mapsto x$ is an isomorphism. The set $\left\{\gamma \in \Gamma_{n}: x(\gamma) \neq 0\right\}$ is called the local support of $x$, which contains lots of information, in particular about rapidly increasing sequences. Suppose that $\left(x_{k}\right)$ is a bounded block sequence. We say that $\left(x_{k}\right)$ has bounded local weight if there exists $j \in \mathbb{N}$ so that each $\gamma$ in the local support of $x_{k}$ has weight at least $m_{j}^{-1}$. We say $\left(x_{k}\right)$ has rapidly decreasing local weight if each $\gamma$ in the local support of $x_{k+1}$ has weight less than $m_{i_{k}}^{-1}$, where $i_{k}=\max \operatorname{ran} x_{k}$. In either case, the sequence $\left(x_{k}\right)$ is an RIS. This allows one to split any bounded block sequence into the sum of rapidly increasing sequences (after passing to subsequence) by splitting the local support. Indeed, given a bounded block sequence $\left(x_{k}\right)$, let $u_{k}$ be the restriction of $x_{k}$ to $\Gamma_{i_{k}}$, where $i_{k}=\max \operatorname{ran} x_{k}$. Write $u_{k}=v_{k}+w_{k}$ inside $\ell_{\infty}\left(\Gamma_{i_{k}}\right)$ as a sum of disjointly supported vectors by splitting the support of $u_{k}$, i.e. the local support of $x_{k}$, according to the weight of the coordinates by choosing some suitable threshold. Let $y_{k}$ and $z_{k}$ be the unique extensions into $\operatorname{span}\left\{d_{\gamma}: \gamma \in \Gamma_{i_{k}}\right\}$ of $v_{k}$ and $w_{k}$ respectively. Then, $x_{k}=y_{k}+z_{k}$ and $\left(y_{k}\right)$ and $\left(z_{k}\right)$ are bounded block sequences. After passing to a subsequence, it is possible to arrange that $\left(y_{k}\right)$ has bounded local weight and $\left(z_{k}\right)$ has rapidly decreasing local weight, and hence both are rapidly increasing sequences. Returning to our operator $T-\lambda$, since it converges to zero on any RIS, it follows that $T-\lambda$ also converges to zero on $\left(x_{k}\right)$, which was an arbitrary bounded block sequence. In particular, $T-\lambda$ is compact.

\section{Other developments and open problems}

We already observed the peculiar properties of $\mathfrak{X}_{A H}$ that follow from the space having very few operators. In particular, it is the first Banach space on which every operator has a nontrivial invariant subspace. Let us now mention another application. In his 1972 memoir, Barry Johnson [25] introduced the notion of amenability of Banach algebras and posed the question of whether the algebra $\mathcal{L}(X)$ of all operators on a Banach space $X$ can ever be amenable. It was pointed out by Garth Dales that the space $\mathfrak{X}_{A H}$ provides the first example simply because $\mathcal{L}\left(\mathfrak{X}_{A H}\right)=\mathcal{K}\left(\mathfrak{X}_{A H}\right) \oplus \mathbb{R}$ Id is the unitisation of $\mathcal{K}\left(\mathfrak{X}_{A H}\right)$, and it was already known that $\mathcal{K}(X)$ is amenable for a $\mathscr{L}_{\infty}$-space $X$.

Since the solution of the scalar-plus-compact problem, a large number of articles have appeared, inspired by the landmark paper of Argyros and Haydon. We briefly mention a few of these. Around the same time that the Argyros-Haydon paper was published, Dan Freeman, Ted Odell and Thomas Schlumprecht [16] obtained another very important result that also uses the Bourgain-Delbaen method of constructing $\mathscr{L}_{\infty^{-}}$ spaces. They show the universality of $\ell_{1}$ as a dual space: if $X$ is a Banach space with separable dual then $X$ embeds into a $\mathscr{L}_{\infty}$-space $Y$ whose dual $Y^{*}$ is isomorphic to $\ell_{1}$. Combining and extending the ideas and techniques in this paper and in the Argyros-Haydon paper led to the following beautiful result of Argyros, Freeman, Haydon, Odell, Raikoftsalis, Schlumprecht and Zisimopoulou: every separable, uniformly convex space is isomorphic to a subspace of a Banach space $X$ with the scalar-plus-compact property and such that $X^{*}$ is isomorphic to $\ell_{1}$. Note that being hereditarily indecomposable, $\mathfrak{X}_{A H}$ has a very limited subspace structure, so this result shows the richness of the class of spaces with the scalar-pluscompact property.

One important open problem mentioned in [4] is whether there is a reflexive Banach space with the scalar-plus-compact property. Such a space would also be the first example of a reflexive space with the invariant subspace property (ISP), i.e. on which every operator has a non-trivial, proper, closed invariant subspace. Although this problem is still open, in a major breakthrough, Argyros and Pavlos Motakis [5] were able to construct the first example of a reflexive space $\mathfrak{X}_{I S P}$ with the invariant subspace property. Moreover, $\mathfrak{X}_{I S P}$ is the first space for which every subspace has the ISP.

We conclude with two further important open problems. As mentioned earlier, for every subspace $Y$ of the GowersMaurey space $\mathfrak{x}_{G M}$, every operator $Y \rightarrow \mathfrak{X}_{G M}$ is a strictly singular perturbation of a scalar multiple of the inclusion operator. It is not known whether there is a space $X$ such that for every subspace $Y$ of $X$, every operator $Y \rightarrow X$ is a compact perturbation of a scalar multiple of the inclusion operator.

We began with a discussion of the most general operator one can construct on a Banach space with the approximation property. For a general Banach space, the Hahn-Banach theorem only guarantees the existence of nuclear operators, which can be written as an absolutely convergent sum of rank-1 operators. The question thus arises whether there is a Banach space $X$ on which every operator is of the form $\lambda \operatorname{Id}+N$, where $N$ is nuclear. As Tim Gowers commented in one of his blogs [20], the Argyros-Haydon space is already 
the 'ultimate space', and this space $X$ would be 'beyond ultimate'.

\section{References}

[1] G. Androulakis and T. Schlumprecht, Strictly singular, non-compact operators exist on the space of Gowers and Maurey, J. London Math. Soc. (2) 64 (2001), no. 3, 655-674. MR1843416

[2] Spiros A. Argyros, A universal property of reflexive hereditarily indecomposable Banach spaces, Proc. Amer. Math. Soc. 129 (2001), no. 11, 3231-3239. MR1844998

[3] S. A. Argyros and V. Felouzis, Interpolating hereditarily indecomposable Banach spaces, J. Amer. Math. Soc. 13 (2000), no. 2, 243-294. MR1750954

[4] Spiros A. Argyros and Richard G. Haydon, A hereditarily indecomposable $\mathcal{L}_{\infty}$-space that solves the scalar-plus-compact problem, Acta Math. 206 (2011), no. 1, 1-54, Doi:10.1007/s11511-011-0058-y. MR2784662 (2012e:46031)

[5] Spiros A. Argyros and Pavlos Motakis, A reflexive hereditarily indecomposable space with the hereditary invariant subspace property, Proc. Lond. Math. Soc. (3) 108 (2014), no. 6, 1381-1416, Doi:10.1112/plms/pdt062. MR3218313

[6] Spiros A. Argyros and Andreas Tolias, Methods in the theory of hereditarily indecomposable Banach spaces, Mem. Amer. Math. Soc. 170 (2004), no. 806, vi+114. MR2053392

[7] S. A. Argyros and A. Tolias, Indecomposability and unconditionality in duality, Geom. Funct. Anal. 14 (2004), no. 2, 247-282. MR2060194

[8] N. Aronszajn and K. T. Smith, Invariant subspaces of completely continuous operators, Ann. of Math. (2) 60 (1954), 345-350, Doi:10.2307/1969637. MR0065807

[9] Stefan Banach, Théorie des opérations linéaires, Z Subwencjyi Funduszu Kultury Narodowej, Warszawa, 1932.

[10] Steven F. Bellenot, Tsirelson superspaces and $l_{p}$, J. Funct. Anal. 69 (1986), no. 2, 207-228. MR865221

[11] J. Bourgain and F. Delbaen, A class of special $\mathscr{L}_{\infty_{\infty}}$ spaces, Acta Math. 145 (1980), no. 3-4, 155-176. MR590288

[12] Per Enflo, A counterexample to the approximation problem in Banach spaces, Acta Math. 130 (1973), 309-317, Doi:10.1007/BF02392270. MR0402468

[13] _ On the invariant subspace problem for Banach spaces, Acta Math. 158 (1987), no. 3-4, 213-313, Doi:10.1007/BF02392260. MR892591

[14] V. Ferenczi, Operators on subspaces of hereditarily indecomposable Banach spaces, Bull. London Math. Soc. 29 (1997), no. 3, 338-344. MR1435570

[15] T. Figiel and W. B. Johnson, A uniformly convex Banach space which contains no $l_{p}$, Compositio Math. 29 (1974), 179-190. MR0355537 (50 \#8011)

[16] D. Freeman, E. Odell, and Th. Schlumprecht, The universality of $\ell_{1}$ as a dual space, Math. Ann. 351 (2011), no. 1, 149-186, Doi:10.1007/s00208-010-0601-8. MR2824850

[17] I. Gasparis, Strictly singular non-compact operators on hereditarily indecomposable Banach spaces, Proc. Amer. Math. Soc. 131 (2003), no. 4, 1181-1189. MR1948110

[18] W. T. Gowers, A solution to Banach's hyperplane problem, Bull. London Math. Soc. 26 (1994), no. 6, 523-530, Doi:10.1112/blms/26.6.523. MR1315601

[19] W. Timothy Gowers, A remark about the scalar-plus-compact problem, Convex geometric analysis (Berkeley, CA, 1996), Math. Sci. Res. Inst. Publ., vol. 34, Cambridge Univ. Press, Cambridge, 1999, pp. 111-115. MR1665582

[20] W. T. Gowers, A remarkable recent result in Banach space theory https://gowers.wordpress.com/2009/02/07/a-remarkable-recent-resultin-banach-space-theory/.

[21] W. T. Gowers and B. Maurey, The unconditional basic sequence problem, J. Amer. Math. Soc. 6 (1993), no. 4, 851-874. MR1201238
[22] Richard Haydon, Subspaces of the Bourgain-Delbaen space, Studia Math. 139 (2000), no. 3, 275-293. MR1762585

[23] _ Variants of the Bourgain-Delbaen construction, Unpublished conference talk, Caceres (2006).

[24] Robert C. James, Uniformly non-square Banach spaces, Ann. of Math. (2) 80 (1964), 542-550. MR0173932

[25] Barry Edward Johnson, Cohomology in Banach algebras, American Mathematical Society, Providence, R.I., 1972. Memoirs of the American Mathematical Society, No. 127. MR0374934

[26] Joram Lindenstrauss, Some open problems in Banach space theory, Séminaire Choquet. Initiation à l'analyse, 15 (1975-76), exp. $n^{\circ} 18$, $1-9$.

[27] B. Maurey and H. P. Rosenthal, Normalized weakly null sequence with no unconditional subsequence, Studia Math. 61 (1977), no. 1, 77-98. MR0438091

[28] C. J. Read, A solution to the invariant subspace problem, Bull. London Math. Soc. 16 (1984), no. 4, 337-401, Doi:10.1112/blms/16.4.337. MR749447

[29] Thomas Schlumprecht, An arbitrarily distortable Banach space, Israel J. Math. 76 (1991), no. 1-2, 81-95. MR1177333

[30] B. S. Cirel'son, It is impossible to imbed $1_{p}$ of $c_{0}$ into an arbitrary Banach space, Funkcional. Anal. i Priložen. 8 (1974), no. 2, 57-60 (Russian). MR0350378 (50 \#2871)

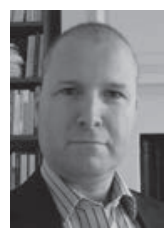

András Zsák [a.zsak@dpmms.cam.ac.uk] is a Fellow of Peterhouse, the oldest college in Cambridge, and affiliated lecturer in the Department of Pure Mathematics and Mathematical Statistics of the University of Cambridge. His research field is the geometry of Banach spaces. 